

\title{
UWB radar detection theory
}

Laila Sakkila, C. Tatkeu, Atika Rivenq, J. Zaidouni, Michael Bocquet

\section{To cite this version:}

Laila Sakkila, C. Tatkeu, Atika Rivenq, J. Zaidouni, Michael Bocquet. UWB radar detection theory. 10th International Symposium on Signal, Image, Video and Communications, ISIVC 2020, postponed event, Apr 2021, Saint-Etienne, France. pp.9487541, 10.1109/ISIVC49222.2021.9487541. hal-03362259

\section{HAL Id: hal-03362259 \\ https://hal.science/hal-03362259}

Submitted on 4 Oct 2021

HAL is a multi-disciplinary open access archive for the deposit and dissemination of scientific research documents, whether they are published or not. The documents may come from teaching and research institutions in France or abroad, or from public or private research centers.
L'archive ouverte pluridisciplinaire HAL, est destinée au dépôt et à la diffusion de documents scientifiques de niveau recherche, publiés ou non, émanant des établissements d'enseignement et de recherche français ou étrangers, des laboratoires publics ou privés. 


\title{
UWB radar detection theory
}

\author{
L. Sakkila ${ }^{1}$, C. Tatkeu ${ }^{2}$, A. Rivenq ${ }^{1}$, J. Zaidouni ${ }^{3}$, and M. Bocquet ${ }^{1}$ \\ Corresponding author: laila.sakkila@uphf.fr
}

\author{
Univ Lille Nord de France, F-59000 Lille, France \\ ${ }^{1}$ IEMN (UMR 8520 CNRS) Dept. OAE, \\ UVHC F- 59313 Valenciennes - France \\ ${ }^{2}$ IFSTTAR-LEOST F-59650 Villeneuve d'Acsq - France \\ ${ }^{3}$ Department of Electronics, Informatics and Telecommunications \\ National School of Applied Sciences Oujda-Morocco
}

\begin{abstract}
In this paper, a study of UWB receivers in terms of detection theory is presented. The UWB radar which is presented in many works previously [1] [2] [3] has many applications. For road UWB radar application, the receiver based on correlation is the optimum receiver [4]. In fact, it maximizes the probability of detection. We will consider, in this study, a correlator receiver based on a threshold detection method.
\end{abstract}

As in narrowband [5] [6], we will describe the theoretical study that evaluates the performance of the UWB receiver based on correlation in terms of detection and false alarm probabilities. Then a study of curves showing threshold receiver operating characteristics (ROC system), based on correlation and destined to be used for a UWB radar is presented. The study is original because it is presented for the first time in a UWB radar system.

Key words-UWB technology, radar, Detection theory, Detection probability, false alarm probability

\section{INTRODUCTION}

$\mathbf{T}$ HE he receiver based on correlation is adapted to detect a ultra-wideband (UWB) radar pulse $s(t)$ of duration $\mathrm{T}$ and auto-correlation $\mathrm{c}(\tau)$, where $\tau$ represents the delay [7]. This auto-correlation function is maximum for zero delay $\tau=0$ and equals 1 if the pulse energy ES is normalized to unity [2].

The received model signal $r(t)$ is a Gaussian white noise $n(t)$ added to the signal. If this signal is present at the receiver input and alone, this case will be called: hypothesis I, otherwise will be called hypothesis II.

$$
r(t)= \begin{cases}s(t)+n(t) & \text { for hypothesis I } \\ n(t) & \text { for hypothesis II }\end{cases}
$$

The noise has zero mean and variance $\sigma^{2}$. The signal to noise ratio $(\mathrm{SNR})$ is given by:

$$
S N R(d B)=10 \log _{10}\left(\frac{E_{s}}{\sigma^{2}}\right)
$$

So:

$$
\sigma=10^{-S N R / 20} \sqrt{E_{s}}
$$

In order to simplify expressions in the following, the energy ES of the signal, in the output of the adapted filter, can be normalized to unity.

The threshold correlator diagram is given in Figure 1

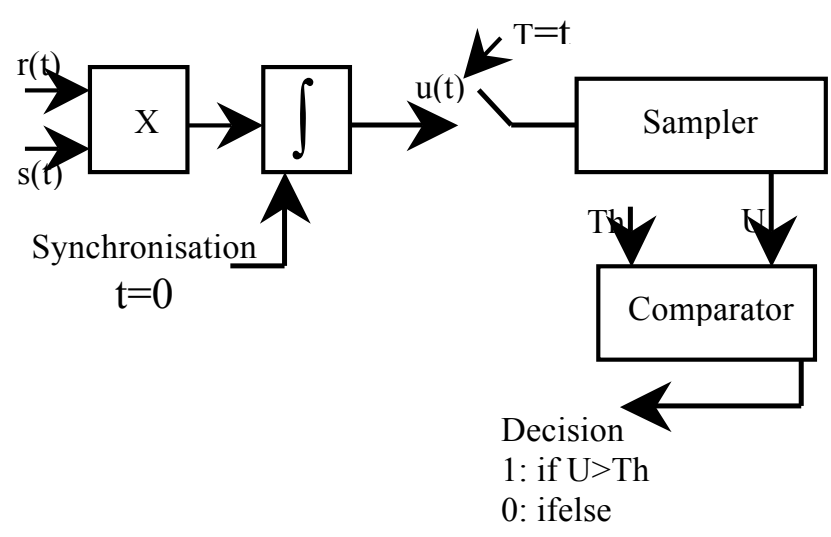

Fig. 1: Threshold correlator implementation

The correlator includes a multiplier, an integrator, a sampler and a comparator (figure 1). First, the received signal $r(t)$ is multiplied by the expected signal $s(t)$, the product is applied to the input of the integrator whose output is written as:

$$
u(t)=\int_{t_{1}=0}^{t} r\left(t_{1}\right) s\left(t_{1}\right) d t_{1}
$$

Note that this implies receiver synchronization at the beginning of the pulse if it is present $(t=0)$.

The sampler digitizes the integrator output at the end of pulse $(\mathrm{t}=\mathrm{T})$, to provide:

$$
U=u(T)=\int_{t_{1}=0}^{T} r\left(t_{1}\right) s\left(t_{1}\right) d t_{1}
$$

We recognize, in this expression, the cross-correlation of $\mathrm{r}(\mathrm{t})$ and $s(t)$ at zero delay $(\tau=0)$. If a synchronization error occurs, 
we obtain the cross-correlation $\mathrm{c}(\tau)$ of $\mathrm{r}(\mathrm{t})$ and $\mathrm{s}(\mathrm{t})$ at nonzero delay $(\tau \neq 0)$

Finally, the comparator allows the comparison between $U$ and a threshold Th: If $\mathrm{U}>\mathrm{Th}$, it is decided that the expected signal is present at the receiver input, otherwise it's absent. This threshold can be adjusted as desired and can take a value either positive, zero or negative.

\section{UWB DETECTION THEORY}

\section{A. Detection Probability}

Suppose that the hypothesis I is true. The signal added to the noise is present at the receiver input.

If we admit the presence of a synchronization error $(\tau)$, we will have then:

$$
U=\int_{t_{1}=0}^{T} s\left(t_{1}\right) s\left(t_{1}+\tau\right) d t_{1}+\int_{t_{1}=0}^{T} s\left(t_{1}\right) n\left(t_{1}+\tau\right) d t_{1}
$$

The first term of this equality is equal to the signal autocorrelation $\mathrm{c}(\tau)$, that is maximum for $\tau=0$ (and equal to unity according to the hypothesis previously supposed $\left.E s=\int s^{2}(t) d t_{1}=1\right)$. This auto-correlation can take also negative values.

The second term is a Gaussian white noise with zero mean and variance $\sigma^{2}$.

So, $\mathrm{U}$ is a Gaussian white noise, with mean $\mathrm{c}(\tau)$ and variance $\sigma^{2}$, and therefore $U$ has the probability density as following[6]:

$$
p_{U(x)}=\frac{1}{\sigma \sqrt{2 \pi}} \exp \left(\frac{-[x-c(\tau)]^{2}}{2 \sigma^{2}}\right)
$$

The probability of detection $P_{D}$ applies to the condition $U>T h$ : the signal is present and the decision taken by the receiver confirms the presence of signal. This is written as:

$$
p_{D}=\frac{1}{\sigma \sqrt{2 \pi}} \int_{T h}^{+\infty} \exp \left(\frac{-[x-c(\tau)]^{2}}{2 \sigma^{2}}\right) d x
$$

Using the function

$$
\operatorname{erfc}(x)=\frac{2}{\sqrt{\pi}} \int_{x}^{+\infty} \exp \left(-t^{2}\right) d t
$$

and putting

$$
t=\frac{[x-c(\tau)]}{(\sigma \sqrt{2})}
$$

We find:

$$
P_{D}=\frac{1}{2} \operatorname{erfc}\left(\frac{T h-c(\tau)}{\sigma \sqrt{2}}\right)
$$

\section{B. False alarm probability}

Suppose the hypothesis II true. So, only noise is present at the receiver input.

If we admit the presence of a synchronization error $(\tau)$, we find:

$$
U=\int_{t_{1}=0}^{T} s\left(t_{1}\right) n\left(t_{1}+\tau\right) d t_{1}
$$

It is a white Gaussian noise of zero mean and variance $\sigma^{2}$, therefore the probability density is:

$$
p_{U}(x)=\frac{1}{\sigma \sqrt{2 \pi}} \exp \left(\frac{-x^{2}}{2 \sigma^{2}}\right)
$$

The false alarm probability $\mathrm{P}_{\mathrm{FA}}$ applies to the case for which the signal is absent but the decision taken by the receiver is for the presence of the signal. This is written as:

$$
p_{F A}=\frac{1}{\sigma \sqrt{2 \pi}} \int_{T h}^{+\infty} \exp \left(\frac{-x^{2}}{2 \sigma^{2}}\right) d x
$$

Using again the fonction erfc $(x)$ and putting $t=x /(\sigma / \sqrt{ } 2)$, we find :

$$
P_{F A}=\frac{1}{2} \operatorname{erfc}\left(\frac{T h}{\sigma \sqrt{2}}\right)
$$

The essential tool of the radar detection theory is the ambiguity function [5] [6]. This function characterizes the signal properties as a measuring instrument. After emission of a known signal and reception of an echo, the ambiguity function measures the similarity of the signal with its translates into time and frequency. It is essentially a measure of correlation according to a delay and a frequency shift.

The radar presented in this paper is a radar based on UWB signals [2]. These UWB signals, with no carrier frequency, do not require study of the ambiguity function.

\section{RECEIVER OPERATIONAL CHARACTERISTICS (ROC SYSTEM)}

The ROC curves represent the detection probability $\mathrm{P}_{\mathrm{D}}$ according to the false alarm probability $\mathrm{P}_{\mathrm{FA}}$ [9]. The parameters controlling the curves are the signal to noise ratio SNR (or standard deviation $\sigma$ ), the synchronization error $\tau$ and the threshold position Th. 
Classically, a curve is obtained for $\tau$ and Th determined, by varying $\sigma$. The set of curves is then obtained by varying Th (and, finally, several sets by varying $\tau$ ).

In fact, all possible representations can be explored first by varying one of the three parameters, the others parameters being fixed (curve), then by varying a second parameter and keeping the third one unchanged (set of curves)), and finally by varying the latter also (all sets).

\section{A. Special cases}

\section{a) High Signal to Noise ratio}

Firstly, suppose the signal to noise ratio (SNR) high, that is to say very low $\sigma$ [8]. The threshold position becomes essential in determining the probabilities $\mathrm{P}_{\mathrm{D}}$ and $\mathrm{P}_{\mathrm{FA}}$.

In the expression (15) of $\mathrm{P}_{\mathrm{FA}}$, the argument of the erfc function tends to $+\infty$ if $\mathrm{Th}>0$ and to $-\infty$ if $\mathrm{Th}<0$. When $\mathrm{Th}=0$, this argument is zero (for small $\sigma$, but not zero). It is therefore:

$$
\lim _{\sigma \rightarrow 0} P_{F A}= \begin{cases}0 & \text { if } T h\rangle 0 \\ \operatorname{erfc}(0) / 2=1 / 2 & \text { if } T h=0 \\ 1 & \text { if } T h\langle 0\end{cases}
$$

Considering the expression (11) of $\mathrm{P}_{\mathrm{D}}$, we see that the argument of the erfc function tends to $+\infty$ if $\mathrm{Th}-\mathrm{c}(\tau)>0$ and to $-\infty$ if $\mathrm{Th}-\mathrm{c}(\tau)<0$. In the case where $\mathrm{Th}=\mathrm{c}(\tau)$ (for $\sigma$ small, but non-zero), the previous argument is zero. It is therefore:

$$
\lim _{\sigma \rightarrow 0} P_{D}= \begin{cases}0 & \text { if } \operatorname{Th}\rangle \mathrm{c}(\tau) \\ \operatorname{erfc}(0) / 2=1 / 2 & \text { if } \operatorname{Th}=\mathrm{c}(\tau) \\ 1 & \text { if } \operatorname{Th}\langle\mathrm{c}(\tau)\end{cases}
$$

\section{b) 2. Low Signal to Noise ratio}

Now suppose the SNR very low, that is to say $\sigma$ high. The position of the threshold becomes almost indifferent to determine the probabilities $\mathrm{P}_{\mathrm{D}}$ and $\mathrm{P}_{\mathrm{FA}}$. In the expressions (15) of $\mathrm{P}_{\mathrm{FA}}$ and (11) of $\mathrm{P}_{\mathrm{D}}$, the argument of the erfc function tends to 0 , so:

$$
\lim _{\sigma \rightarrow \infty} P_{F A}=\lim _{\sigma \rightarrow \infty} P_{D}=\operatorname{erfc}(0) / 2=1 / 2
$$

\section{c) Median threshold}

Now, consider the case of a median threshold, such as the values of Th and Th $-\mathrm{c}(\tau)$ is symmetrical about 0 , that is to say $\mathrm{Th}=\mathrm{c}(\tau) / 2$. This corresponds to the line, in the set of curves in Figure 2, passing through the points $(0,1)$ and $(1 / 2,1 / 2)$. The expressions (15) of $\mathrm{P}_{\mathrm{FA}}$ and (11) of $\mathrm{P}_{\mathrm{D}}$ become:

$$
\begin{aligned}
P_{D} & =\frac{1}{2} \operatorname{erfc}\left(\frac{-c(\tau)}{2 \sigma \sqrt{2}}\right) \\
& =\frac{1}{2} \operatorname{erfc}(0)+\frac{1}{\sqrt{\pi}} \int_{-c(\tau) /(2 \sigma \sqrt{2})}^{0} \exp \left(-t^{2}\right) d t \\
& =\frac{1}{2}+\frac{1}{\sqrt{\pi}} \int_{0}^{c(\tau) /(2 \sigma \sqrt{2})} \exp \left(-t^{2}\right) d t
\end{aligned}
$$

$$
\begin{aligned}
P_{F A} & =\frac{1}{2} \operatorname{erfc}\left(\frac{c(\tau)}{2 \sigma \sqrt{2}}\right) \\
& =\frac{1}{2} \operatorname{erfc}(0)-\frac{1}{\sqrt{\pi}} \int_{0}^{c(\tau) /(2 \sigma \sqrt{2})} \exp \left(-t^{2}\right) d t \\
& =\frac{1}{2}-\frac{1}{\sqrt{\pi}} \int_{0}^{c(\tau) /(2 \sigma \sqrt{2})} \exp \left(-t^{2}\right) d t
\end{aligned}
$$

From equations (19) and (20), we deduce that:

$$
\mathrm{P}_{\mathrm{D}}+\mathrm{P}_{\mathrm{FA}}=1
$$
a line.

For Th and $\tau$ fixed (so only $\sigma$ varies), it is the equation of

Moreover, if we suppose $\mathrm{c}(\tau)>0$, we see that $\mathrm{P}_{\mathrm{D}}>1 / 2$ and $\mathrm{P}_{\mathrm{FA}}<1 / 2$.

\section{d) High threshold}

Now, consider the case of a high threshold as $\mathrm{Th}=\mathrm{c}(\tau)$ (we suppose that $\mathrm{c}(\tau)>0)$. The expressions 15 of $\mathrm{P}_{\mathrm{FA}}$ and 11 of $\mathrm{P}_{\mathrm{D}}$ become:

$$
\begin{aligned}
& P_{D}=\frac{1}{2} \operatorname{erfc}(0)=\frac{1}{2} \\
& P_{F A}=\frac{1}{2} \operatorname{erfc}\left(\frac{c(\tau)}{2 \sigma \sqrt{2}}\right)
\end{aligned}
$$

From the above equation, we deduce that $\mathrm{P}_{\mathrm{FA}}<1 / 2$ if $\mathrm{c}(\tau)<0$.

\section{e) Low threshold}

Finally, consider the case of a low threshold, such that $\mathrm{Th}=0$. The expressions (15) of $\mathrm{P}_{\mathrm{FA}}$ and (11) of $\mathrm{P}_{\mathrm{D}}$ become:

$$
\begin{aligned}
P_{D} & =\frac{1}{2} \operatorname{erfc}\left(\frac{-c(\tau)}{2 \sigma \sqrt{2}}\right) \\
& =\frac{1}{2}+\frac{1}{\sqrt{\pi}} \int_{0}^{c(\tau) /(2 \sigma \sqrt{2})} \exp \left(-t^{2}\right) d t
\end{aligned}
$$




$$
P_{F A}=\frac{1}{2} \operatorname{erfc}(0)=\frac{1}{2}
$$

The first equation allows concluding that $P_{D}>1 / 2$ if we suppose also that $\mathrm{c}(\tau)>0$.

\section{B. Study Procedure}

It is easier to ignore the parameter $\tau$ (synchronization error) at the first time. Reference ROC Networks are so established to zero delay (for $\tau=0$ that to say $\mathrm{c}(\tau)=1$ ).

\section{a) References ROC networks}

We assume therefore $\mathrm{c}(\tau)=1$ and there are two parameters SNR and Th. We can then draw two distinct sets of curves. In the first set, each curve corresponds to a fixed value of Th and is described by varying SNR (thus $\sigma$ ). For the second set, the roles of SNR and Th are exchanged.

The sets can be exploited by identifying areas of the plane (SNR, Th) (or $(\sigma, \mathrm{Th})$ ), said exploitable, in which the detection probability is greater than some minimum limit, the false alarm probability being below a limit fixed:

$$
\begin{aligned}
& \left.P_{D}\right\rangle P_{D}^{\min } \\
& \left.P_{F A}\right\rangle P_{F A}^{\min }
\end{aligned}
$$

\section{b) Influence of $\tau$}

Precedent reference sets and working zones can be used for any value of $\tau$. The method is explained below for $\mathrm{c}(\tau)>0$.

An effective signal to noise ratio and an apparent threshold are defined, which allow using ROC reference sets of curves. In fact, the SNR is decreased compared to its real value, because that $\mathrm{c}(\tau)<1$. The apparent SNR is given by:

$$
\mathrm{SNR}^{\prime}=\mathrm{SNR}-10 \log 10[\mathrm{c}(\tau)]
$$

However, the threshold appears increased compared to its real value:

$$
\mathrm{Th}^{\prime}=\mathrm{Th} / \mathrm{c}(\tau)
$$

The working zones can then be used to determine the default value of the tolerable synchronization.

\section{RESUlTS}

The reference ROC curves have been established using the Matlab software.
Figure 2 shows the ROC curves obtained by taking Th as the parameter. Each curve corresponds to a particular value of SNR.

ROC curves (parameter threshold "Th")

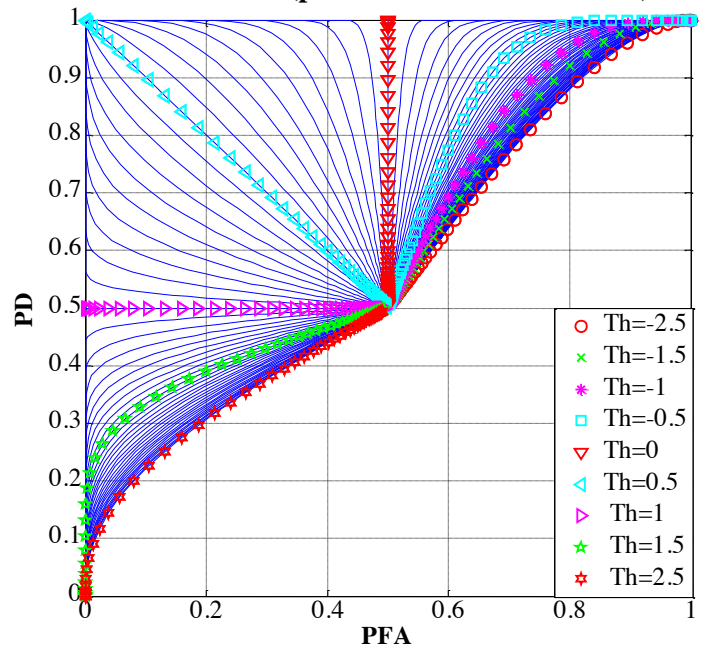

Fig 2: ROC curves for $\mathrm{c}(\tau)=1-$ Parameter Th.

Figure 3 represents the ROC curves obtained by taking SNR as the parameter. Each curve corresponds to a particular value of Th.

It may be noted that the corresponding curves are orthogonal to those of Figure 2.

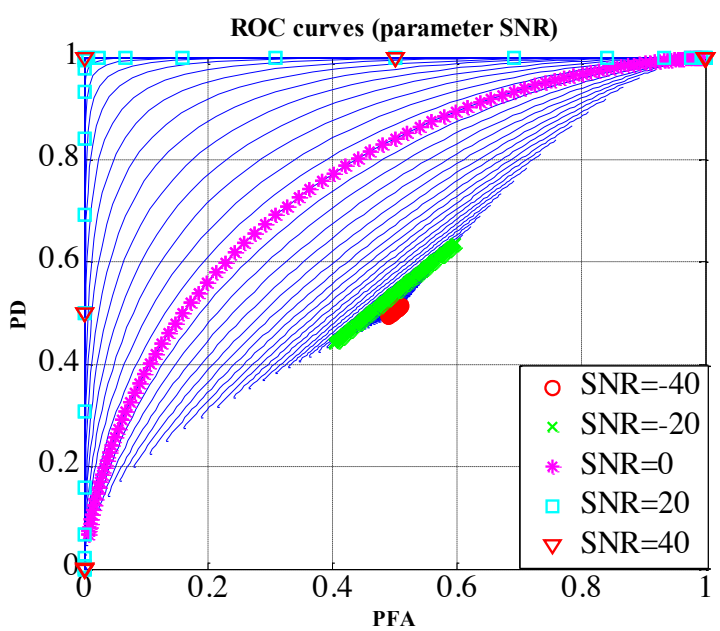

Fig. 3: ROC curves for $\mathrm{c}(\tau)=1-$ Parameter SNR.

Figure 4 provides the working zone in plane (SNR, Th) where the detection probability is above $99 \%$ and the false alarm less than $1 \%$. 


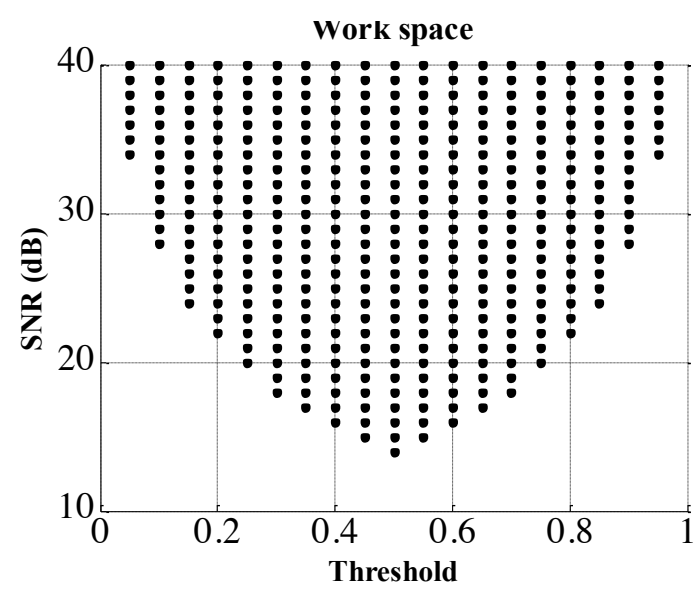

Fig 4: Exploitable area for $\mathrm{P}_{\mathrm{D}}>99 \%$ and $\mathrm{P}_{\mathrm{FA}}<1 \%$.

\section{CONCLUSION}

A radar reception stage is characterized using the theory of detection of its receiver. In this paper, the correlation receiver detection theory of UWB radar has been presented. This study has ever been conducted for a UWB radar, where the originality of the work presented in this paper. The performance of the correlation receiver for UWB radar has been theoretically evaluated in terms of detection probability and false alarm probability. Following these results set of ROC curves were obtained. Several cases from the SNR and threshold have been treated. Finally a working zone which allows a detection probability greater than $99 \%$ and a probability of false alarm less than $1 \%$ has been provided

\section{REFERENCES}

[1] L. Sakkila "Short Range radar based on UWB technology," Radar technolgy, IN-TECH, ISBN 978-953-307-029-2, Edited by Guy Kouemou, published December 2009.

[2] L. Sakkila « "Study and implementation of an ultra wideband radar for detection and recognition of obstacles Road environment "PhD thesis at the University of Valenciennes, December 2009

[3] L. Sakkila "UWB short range radar for road applications" Phys. Chem. News, 64 (2012) 20-29 (published march 2012).

[4] Y. Kwon, R. Narayanan, and M. Rangaswamy, "Multi-target detection using total correlation for noise radar systems," Aerospace and Electronic Systems, IEEE Transactions on, vol. 49, pp. 1251-1262, APRIL 2013.

[5]: P. Woodward, « Probability and Information Theory with Applications to Radar», Artech House, 1980

[6] P. traduit par J. Maurein, "Probabilités, analyse fréquentielle, information, théorie du radar," Eyrolles, Paris, 1980.

[7] C.-P. Lai and R.M.Narayanan, "Ultrawideband random noise radar design for through-wall surveillance," Aerospace and Electronic Systems, IEEE Transactions on, vol. 46, no. 4, pp. 1716-1730, 2010.

[8] L.Sakkila, P.Deloof, Y.Elhillali, A.Rivenq, and S.Niar, "A real time signal processing for an anticollision road radar system," Vehicular Technology Conference, 2006. VTC-2006 Fall. 2006 IEEE 64th, pp. 1-5, September 2006.
[9] J. Zaidouni, A. Rivenq-Menhaj, K. Ghoumid, A. El Moussati, and P. Deloof, "Roc performances of constant false alarm coded anticollision radar in multiuser environment," International Journal of Wireless Information Networks, vol. 22 , no. 4, pp. $369-38$ 